\title{
Development of high drug-loading nanomicelles targeting steroids to the brain
}

This article was published in the following Dove Press journal:

International Journal of Nanomedicine

17 December 2013

Number of times this article has been viewed

\author{
Sijia Zhengl,* \\ Yanqi Xie ${ }^{1, *}$ \\ Yuan $\mathrm{Li}^{1}$ \\ Ling $\mathrm{Li}^{\prime}$ \\ Ning Tian' \\ Wenbo Zhu' \\ Guangmei Yan ${ }^{2}$ \\ Chuanbin $\mathrm{Wu}^{\prime}$ \\ Haiyan $\mathrm{Hu}^{\prime}$ \\ 'School of Pharmaceutical Sciences, \\ 2Department of Pharmacology, \\ Zhongshan School of Medicine, \\ Sun Yat-sen University, Guangzhou, \\ People's Republic of China \\ *These authors contributed equally \\ to this work
}

\begin{abstract}
The objective of this research was to develop and evaluate high drug-loading ligand-modified nanomicelles to deliver a steroidal compound to the brain. YC1 (5 $\alpha$-cholestane24-methylene-3 $\beta, 5 \alpha, 6 \beta, 19$-tetraol), with poor solubility and limited access to the brain, for the first time, has been proved to be an effective neuroprotective steroid by our previous studies. Based on the principle of 'like dissolves like', cholesterol, which shares the same steroidal parent nucleus with YC1, was selected to react with sodium alginate, producing amphiphilic sodium alginatecholesterol derivatives (SACDs). To increase the grafting ratio and drug loading, cholesterol was converted to cholesteryl chloroformate, for the first time, before reacting with sodium alginate. Further, lactoferrin was conjugated on SACDs to provide lactoferrin-SACDs (Lf-SACD), which was established by immune electron microscopy (IEM) and self-assembled into brain-targeting nanomicelles. These nanomicelles were negatively charged and spherical in nature, with an average size of $<200 \mathrm{~nm}$. The YC1 drug loading was increased due to the cholesteryl inner cores of the nanomicelles, and the higher the grafting ratio was, the lower the critical micelle concentration (CMC) value of SACD, and the higher drug loading. The in vitro drug release, studied by bulkequilibrium dialysis in $20 \mathrm{~mL}$ of $6 \%$ hydroxypropyl- $\beta$-cyclodextrin solution at $37^{\circ} \mathrm{C}$, indicated a prolonged release profile. The YC1 concentration in mouse brain delivered by lactoferrin-modified nanomicelles was higher than in those delivered by non-modified nanomicelles and YC1 solution. The unique brain-targeting nanomicelle system may provide a promising carrier to deliver hydrophobic drugs across the blood-brain barrier for the treatment of brain diseases.
\end{abstract}

Keywords: drug-loading, lactoferrin, nanomicelles, sodium alginate, cholesterol, braintargeting

\section{Introduction}

YC1 ( $5 \alpha$-cholestane-24-methylene-3 $\beta, 5 \alpha, 6 \beta, 19$-tetraol) (Figure 1) is a kind of oxysterol extracted for the first time from the soft coral Nephthea brassica in the South Sea of China. ${ }^{1}$ Our previous studies have demonstrated that $\mathrm{YC} 1$ plays a significant role in protecting neurons. ${ }^{2}$ However, it was found that the neuroprotection of YC1 after intravenous injection was substantially impeded by its poor solubility and nonspecific systemic distribution. Therefore, it is meaningful and necessary to develop an alternative brain-targeting delivery system of YC1 for its beneficial applications.

Recently, self-assembling micelles as promising drug delivery systems have been paid increasing attention on account of their uncomplicated preparing conditions and relatively high drug loading. ${ }^{3,4}$ According to the empirical rule of 'like dissolves like' (specifically, the greater the similarity between molecular structures, the higher the mutual solubility due to the stronger intermolecular attractions), ${ }^{5}$ if the inner core of nanomicelles presents
Correspondence: Chuanbin Wu; Haiyan $\mathrm{Hu}$

Tel +860203934 3II8

Fax +8602039343118

Email cbwu2000@yahoo.com;

Isshhy@mail.sysu.edu.cn
International Journal of Nanomedicine 2014:9 55-66

Dovepress

http://dx.doi.org// 0.2147/IJN.S52576 (c) (1) () 2014 Zheng et al. This work is published by Dove Medical Press Limited, and licensed under Creative Commons Attribution - Non Commercial (unported, v3.0) License. The full terms of the License are available at http://creativecommons.org/licenses/by-nc/3.0/. Non-commercial uses of the work are permitted without any further permison how to request permission may be found at: http://www.dovepress.com/permissions.php 


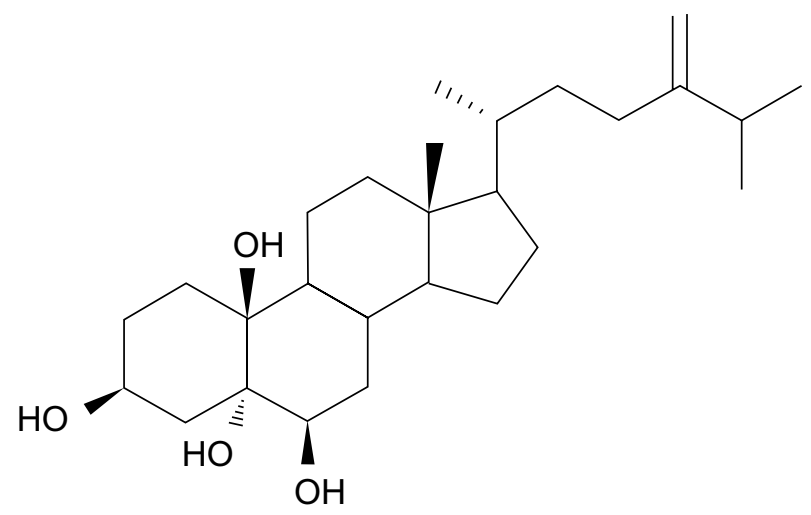

Figure I Chemical structure of $\mathrm{YCI}(5 \alpha$-cholestane-24-methylene- $3 \beta, 5 \alpha, 6 \beta$, 19-tetraol).

a similar structure to the drug to be loaded, then a high drug loading can be expected. Alginates, which are hydrophilic linear anionic polysaccharides, ${ }^{6}$ are commonly used in biotechnological and pharmaceutical fields due to their favorable biocompatibility, biodegradability, non-toxicity, and nonimmunogenicity. ${ }^{7}$ If steroidal cholesterol was conjugated with alginates to produce amphiphilic sodium alginate-cholesterol derivatives (SACDs), the inner cores of the obtained nanomicelles would be full of cholesteryl, which is expected to strongly absorb the steroidal YC1. Thus, nanomicelles with relatively high drug loading of YC1 could be prepared.

Another obstacle that strongly contains the neuroprotection of $\mathrm{YC} 1$ is the blood-brain barrier (BBB), which prevents the cerebral uptake of over $98 \%$ of small molecule drugs and $100 \%$ of large molecule drugs. ${ }^{8}$ Consequently, a large number of newly discovered drugs, including $\mathrm{YC} 1$, lose their value of development due to poor permeability through the BBB. ${ }^{9}$ In this regard, developing brain-targeting drug delivery systems for $\mathrm{YC} 1$ to circumvent this permeability challenge is urgent.

Fortunately, the BBB possesses a special receptormediated endocytosis mechanism that may provide an effective approach to targeting medications to the brain., ${ }^{4,10}$ Currently, the specific receptors identified on the BBB mainly encompass insulin receptors, insulin-like growth factor receptors, epidermal growth factor receptors, folic acid receptors, and transferrin receptors (TfRs). ${ }^{11,12}$ Amongst these receptors, only the insulin receptors and TfRs have been expounded. ${ }^{4,13}$ Since the pharmacological effect of insulin is indispensable and the expression of TfRs is limited to brain capillaries, ${ }^{14}$ it seems logical to choose TfRs as the specific delivery site for brain-targeting. However, the plasma concentration of endogenous Tf is so high that the TfRs are almost saturated under the physiologic condition. ${ }^{15}$ Thus, TfRs are limited to be utilized in brain-targeting drug transportation.
Lactoferrin (Lf), one of the Tf family members, is a single-chain, iron-binding glycoprotein with a molecular weight (MW) of $80 \mathrm{kDa},{ }^{16}$ which has several particular advantages as a brain-targeting ligand compared with Tf. First, the plasma concentration of $\operatorname{Lf}(238.0 \mathrm{ng} / \mathrm{mL})$ is much lower than the saturation value of Lf receptors (LfRs) in the BBB. ${ }^{17,18}$ Second, thanks to its higher affinity with iron, Lf, by binding with iron as a complex, may cross the bovine brain capillary endothelial cells (BBCECs) more easily. ${ }^{19,20}$ Third, Lf is capable of traversing the BBCECs from the apical side to the basolateral side, suggesting that LfRs may be able to mediate more chemicals across the BBB. ${ }^{19}$ Moreover, Lf participates in the pathogenesis of brain lesions. It has been reported that the expression of LfRs increased in patients with Parkinson's disease. ${ }^{21}$ More importantly, a marked elevation of $\mathrm{Lf}$ in the cerebrospinal fluid (CSF) of patients with acute stroke was observed. ${ }^{22}$ In another study, the enhanced endocytotic activity of Lf was revealed in a rat ischemia/stroke model in which transient focal ischemia was induced by using the filament occlusion of middle cerebral arteries. ${ }^{23}$ Therefore, it was concluded that such an increase in transcytosis of $\mathrm{Lf}$ did not involve the upregulation of the LfRs, but rather an increase of transcytosis transport. ${ }^{24}$ Accordingly, these findings suggested that Lf may be a promising brain-targeting ligand that could significantly enhance $\mathrm{YC} 1$, a potential therapeutic agent for central nervous system (CNS) disorders, to penetrate the BBB.

In this research, Lf-SACDs were synthesized and then self-assembling nanomicelles were prepared for loading and targeting delivery of YC1 to the brain. The hydrophobic cholesteryl inner core of the nanomicelles may increase the drug loading of YC1 owing to the structural similarity between cholesterol and YC1. Meanwhile, the Lf conjugated on the hydrophilic outer layer may enhance the LfRs-mediated endocytosis of nanomicelles, generating an elevation of YC1 cerebral distribution. The emulsification method was used to encapsulate YC1 into the nanomicelles. The particle size, zeta potential, morphology, grafting ratio, encapsulation efficiency, and the in vitro releasing profile of the YC1-loaded nanomicelles were studied. The brain-targeting activity of the YC1-loaded Lf-SACD nanomicelles was evaluated in vivo. The distribution of YC1 in major organs was characterized and the targeting parameters were calculated.

\section{Materials and methods Materials}

Sodium alginate (Protanal LF 120M with MW of $180-250 \mathrm{kDa}$ ) containing $35 \%-45 \%$ of guluronic acid and 
$55 \%-65 \%$ of mannuronic acid was a kind gift from FMC BioPolymer (Philadelphia, PA, USA). Formamide (FA) and dimethyl formamide (DMF) were dried by molecular sieve. $p$-toluenesulfonic acid (pTSA) was dried at $80^{\circ} \mathrm{C}$ for 20 minutes in a vacuum. Cholesterol was bought from Qi Hua Armamentarium (Guangzhou, People's Republic of China). The internal standard (ST009, $5 \alpha$-androstane- $3 \beta$, 5, 6ß-triol), lactoferrin (Lf, from bovine), dicyclohexylcarbodiimide (DCC), $N$-hydroxysulfosuccinimide (NHSS), 1-ethyl-3-[3-dimethylaminopropyl] carbodiimide hydrochloride (EDC), 4-( $N, N^{\prime}$-dimethylamino)pyridine (DMAP), and 2-(4-morpholino) ethanesulfonic acid (MES) were purchased from Sigma-Aldrich (St Louis, MO, USA) and used without further purification. The anti-Lf antibody was a rabbit polyclonal antibody raised against bovine. Hydroxypropyl$\beta$-cyclodextrin (HP- $\beta$-CD) was provided by Roquette (Lestrem, France). All the other chemicals and solvents were of chromatographic and pharmaceutical grade.

\section{Synthesis and characterization of SACD}

Cholesterol $(0.66 \mathrm{~g})$ and triphosgene $(1.00 \mathrm{~g})$ were reacted in tetrahydrofuran (THF) at $40^{\circ} \mathrm{C}$ for 30 minutes, ${ }^{25}$ and only one hydroxyl of cholesterol was converted into cholesteryl chloroformate. Then, according to Yang et al, ${ }^{7}$ water-free pTSA $(0.32 \mathrm{~g})$ and sodium alginate $(1.00 \mathrm{~g})$ were dispersed in a mixture of $38 \mathrm{~mL}$ of FA/DMF $(10: 9, \mathrm{v} / \mathrm{v})$ in a $250 \mathrm{~mL}$ round bottom flask, and the sodium alginate was partially hydrolyzed into alginic acid at $50^{\circ} \mathrm{C}$ by stirring for 30 minutes. DCC $(0.40 \mathrm{~g})$, DMAP $(0.48 \mathrm{~g})$, and the solution of chloroform containing activated cholesteryl chloroformate were then added to the suspension of sodium alginate. The reaction between alginic acid and the chloroformate of cholesterol was carried out at $25^{\circ} \mathrm{C}-30^{\circ} \mathrm{C}$ by stirring for 24 hours. The product was purified with $200 \mathrm{~mL}$ of ethanol, with stirring at $40^{\circ} \mathrm{C}$, and then centrifuged. The obtained pellets were dried under vacuum for 24 hours at $40^{\circ} \mathrm{C}$, dissolved in distilled water, and neutralized by adding the solution of $1.5 \%$ $\mathrm{Na}_{2} \mathrm{CO}_{3}$. The obtained solution was dialyzed for 3 days and further freeze-dried to collect SACD.

The ${ }^{1} \mathrm{H}$ NMR spectra of SACD were studied by ${ }^{1} \mathrm{H}$ nuclear magnetic resonance spectroscopy (Avance III 400 Plus; Bruker, Billerica, MA, USA); FT-IR measurement was carried out on a Fourier transform infra-red spectrometer (Tensor37, Bruker) at $0.5 \mathrm{~cm}^{-1}$ using $\mathrm{KBr}$ pellets.

\section{Synthesis of Lf-SACD}

Lactoferrin was conjugated to SACD through the carboxylic acid groups of alginic acid. According to Liu et al, ${ }^{26}$
100 and $40 \mathrm{mM}$ solutions of EDC and NHSS were prepared using 0.1 M MES solution, respectively. SACD was added to the $0.1 \mathrm{M}$ MES $(0.5 \mathrm{~mL})$ with $40 \mu \mathrm{L}$ of EDC and NHSS, and the obtained solution was incubated at $4{ }^{\circ} \mathrm{C}$ for 1 hour. Then, Lf was dissolved into phosphate-buffered saline (PBS, $\mathrm{pH} 7.4$ ) to form a $1.0 \mathrm{mg} / \mathrm{mL}$ solution, and the activated SACD $(5.0 \mathrm{mg})$ solution and Lf solution $(125 \mu \mathrm{L})$ were reacted for 24 hours at room temperature. The product was dialyzed against distilled water for 3 days using the Spectra/Pro membrane with a molecular weight cut-off of $100 \mathrm{kDa}$ and the final product was obtained after lyophilization.

\section{Characterization of Lf-SACD}

Immune electron microscopy (IEM) was harnessed to confirm the successful conjugation of Lf to SACD. Briefly, Lf $(0.13 \mathrm{mg})$, SACD $(1.2 \mathrm{mg})$, and Lf-SACD $(1.2 \mathrm{mg})$ were separated by $10 \%$ SDS-PAGE. After electrophoresis, the proteins were electroblotted to a polyvinylidene fluoride (PVDF) membrane $(0.45 \mu \mathrm{m}$; Millipore, Billerica, MA, USA). The PVDF membrane was then blocked with 5\% skimmed milk (Bio-Rad, Hercules, CA, USA) in Tris HClbuffered saline solution (TBS, $0.1 \mathrm{~g} \mathrm{KCl}, 4.0 \mathrm{~g} \mathrm{NaCl}$, and $1.5 \mathrm{~g}$ Tris, $\mathrm{pH}$ 7.4) for 2 hours. Lf antibodies were added and the system was incubated for 2 hours, and then reacted with the rabbit antibodies for 1 hour. The immune complexes were visualized using the electrochemiluminescence (ECL) detection reagents.

\section{Preparation of self-aggregating nanomicelles}

A microphase separation method was used to prepare nanomicelles. Briefly, $20.0 \mathrm{mg}$ of SACD and $8.0 \mathrm{mg}$ of $\mathrm{YC} 1$ were dispersed into $10 \mathrm{~mL}$ of tetrahydrofuran (THF), respectively, and then added to the distilled water $(10 \mathrm{~mL})$ drop-by-drop under stirring until THF was completely volatilized. ${ }^{27}$ Subsequently, YC1-SACD nanomicelles were formed. Lf-SACD nanomicelles were generated by a similar procedure.

\section{Characterization of the blank and $\mathrm{YCl}$-loaded nanomicelles}

Micelle size and zeta potential

The average size and zeta potential of nanomicelles were determined by Zetasizer Nano ZS90 (Malvern Instruments Ltd, Malvern, UK). The concentration of the nanomicelles was about $1.0 \mathrm{mg} / \mathrm{mL}$ in distilled water. ${ }^{28}$ 


\section{Analysis of morphology by transmission} electron microscope

The morphology of YC1-loaded and YC-unloaded nanomicelles was observed using transmission electron microscopy (TEM, JEM2010 frozen type; JEOL, Tokyo, Japan). The sample was filtrated through a $0.45 \mu \mathrm{m}$ Millipore membrane filter, and then $40 \mu \mathrm{L}$ of the obtained filtrate was placed on a copper chip, which was exposed to air to dry at room temperature and then mounted on the TEM for observation.

\section{Critical micelle concentration of SACD}

The critical micelle concentration (CMC) of SACD was estimated by spectrofluorophotometer (LS55; Perkin Elmer, Waltham, MA, USA), using pyrene as a fluorescence probe. The excitation wavelength was $334 \mathrm{~nm}$ and the slit width was $10 \mathrm{~nm}$. The fluorescence emission spectra were chosen between $360-500 \mathrm{~nm}$ with a slit width of $2.5 \mathrm{~nm}$. SACD was dissolved in $0.15 \mathrm{~mol} / \mathrm{L}$ of $\mathrm{NaCl}$ solution to form a concentration range from 9.0 to $1.0 \times 10^{-3} \mathrm{mg} / \mathrm{mL}$. Pyrene (Acros Organics, Geel, Belgium) was added to the SACD solutions to obtain the pyrene concentration of $2 \mu \mathrm{mol} / \mathrm{L}$. All samples were treated by ultrasonic sound to make sure the pyrene was completely embedded into the hydrophobic microdomains. ${ }^{7}$ The intensity ratios of $\mathrm{I}_{373} / \mathrm{I}_{384}$ were plotted as a function of logarithm concentration of the polymers. ${ }^{29} \mathrm{The} \mathrm{CMC}$ value was taken from the intersection of the tangent to the curve at the inflection with the horizontal tangent through the points at low concentrations.

\section{Determination of grafting ratio}

As described above, after 24 hours reaction of cholesterol and sodium alginate, $200 \mathrm{~mL}$ of ethanol was added to the solution to extract the unreacted cholesterol. After centrifugation, the volume of the supernatant was determined and diluted to an appropriate concentration to be measured by high performance liquid chromatography (HPLC, 1200; Agilent, Santa Clara, CA, USA) with an ODS column (Phenomenex, $250 \times 4.6 \mathrm{~mm}, 5 \mu \mathrm{m}$ ) held in an oven at $38^{\circ} \mathrm{C}$. The mobile phase consisted of methanol with a flow rate of $1.0 \mathrm{~mL} /$ minute. The ultraviolet detecting wavelength was $205 \mathrm{~nm}$ and the injection volume was $10 \mu \mathrm{L}$. The grafting ratio was calculated using the following formula:

Grafting ratio $(\%)=($ amount of total cholesterol added to the reaction system) - (amount of cholesterol in supernatant)/total amount of sodium alginate $\times 100$

\section{Measurement of encapsulation efficiency and drug loading}

The encapsulated and unencapsulated YC1 were separated using a column of Sephadex G-50 (Amersham Biosciences, Uppsala, Sweden). Sephadex G-50 was pretreated and packed into a $5 \mathrm{~mL}$ syringe. Distilled water went through the syringe at a rate of $0.2 \mathrm{~mL} /$ minute for 1 hour to remove air bubbles. The sample was then loaded into the column, with distilled water as the mobile phase at a flow rate of $1.0 \mathrm{~mL} /$ minute. Eluate from 0-16 minutes was collected and the concentrations were determined by liquid chromatography coupled with mass spectrometry (LC-MS, 6120; Agilent). The LC column was Agilent XDB-C18 $(50 \times 4.60 \mathrm{~mm}, 1.8 \mu \mathrm{m})$. The mobile phase, consisting of methanol/water $(90 / 10, \mathrm{v} / \mathrm{v})$, was delivered at a flow rate of $0.5 \mathrm{~mL} /$ minute. The column oven temperature was maintained at $30^{\circ} \mathrm{C}$ and the injection volume was $1.0 \mu \mathrm{L}$. The Agilent's single quadrupole mass spectrometer (6120 series) was equipped with an atmospheric pressure chemical ionization (APCI) source. At first, the full scanning mode was used to select the characteristic ions to be monitored for YC1 and the internal standard (ST009). Next, selective ion monitoring (SIM) was used to monitor the molecular weight (MW) of $403.3 \mathrm{~g} / \mathrm{mol}$ for YC1 and $273.1 \mathrm{~g} / \mathrm{mol}$ for ST009. The standard curve was constructed by dissolving $\mathrm{YCl}$ in methanol at the concentration range of $1.0-25 \mu \mathrm{g} / \mathrm{mL}$ with an $r^{2}$ value of 0.9996 . Encapsulation efficiency $(\%)=$ weight of YC1 measured in total eluate/weight of total YC1 $\times 100$; drug loading $(\%)=$ weight of YC1 measured in total eluate/weight of the blank nanomicelles.

\section{In vitro drug release}

First, the solubility of $\mathrm{YC} 1$ in different solvents was determined and $6 \% \mathrm{HP}-\beta-\mathrm{CD}$ solution was proved to be the most effective solvent among 30\% alcohol, $40 \%$ alcohol, $50 \%$ alcohol, $1 \%$ Tween $80,2 \%$ Tween $80,1 \%$ Tween 80 plus $50 \%$ alcohol, $4 \%$ HP- $\beta-C D$, and $6 \%$ HP- $\beta-C D$.

Second, YC1-loaded nanomicelles $(2.0 \mathrm{mg})$ purified by the Sephadex G-50 column were dissolved in $2.0 \mathrm{~mL}$ of distilled water. The solution was added to the dialysis membrane bag with a molecular weight cut-off of $14 \mathrm{kDa}$. The membrane bag was then immersed into $20 \mathrm{~mL}$ of $6 \%$ HP- $\beta-C D$ solution at a shaking rate of 100 times per minute at $37^{\circ} \mathrm{C}$. A volume of $500 \mu \mathrm{L}$ release medium was taken at $0,0.25,0.5,1,2,4,6,8,10,12$, and 24 hours, respectively, and immediately replaced with the same volume of fresh release medium. The $\mathrm{YC} 1$ content in the release medium was determined by LC-MS as mentioned above. Each experiment was repeated in triplicate. The release rate was calculated 
with the formula: $\mathrm{RR}=\left(W_{i} / W_{\text {total }}\right) \times 100 \%$, where $\mathrm{RR}$ is the drug release rate (\%), $W_{i}$ is the measured amount of $\mathrm{YC} 1$ at the time point of $i$ th hour in the release medium, and $W_{\text {total }}$ is the total amount of YC1 in the equal volume of nanomicelle suspensions prior to dialysis. The release characteristics were explored by the similar factor method, the zero grade model, the first grade model, the Higuchi model, and the Regter-Peppas model.

\section{Biodistribution of $\mathrm{YCI}$ in vivo}

All procedures involving animals were approved by the Institutional Authority for Laboratory Animal Care and Ethics Committee. Adult Kunming mice of either sex (1822 g, Laboratorial Animal Center, Sun Yat-sen University, Guangzhou, People's Republic of China) were injected with Lf-modified nanomicelles (LF group), SACD nanomicelles without modification (MC group), and YC1 solution (YC group) via the tail vein at a dose of $8 \mathrm{mg} / \mathrm{kg}$, respectively. The mice were anesthetized by inhalation of diethyl ether before blood sample collection.

At 15 minutes, 30 minutes, 1 hour, 2 hours, 4 hours, and 8 hours, blood samples were collected from the ocular artery directly after eyeball removal and placed into test tubes containing sodium citrate. Plasma was immediately separated by centrifugation. After that, the mice were sacrificed and the brains, hearts, livers, lungs, and kidneys were excised. The organs were rinsed three times in physiological saline $(0.9 \%$ $\mathrm{NaCl}$ solution) to wash away the residual blood, blotted on filter paper, weighed, and then homogenized in a 3-fold volume $(\mathrm{m} / \mathrm{v})$ of saline. The separated plasma and the organ homogenates were stored at $-20^{\circ} \mathrm{C}$ until assay.

Acetic ether $(5 \mathrm{~mL}), 0.12 \mathrm{mg} / \mathrm{mL} \mathrm{ST009}(10 \mu \mathrm{L})$ as the internal standard solution, $20.3 \mathrm{mg} / \mathrm{mL}$ EDTA (ethylene diamine tetraacetic acid, $10 \mu \mathrm{L}$ ), and $20.9 \mathrm{mg} / \mathrm{mL}$ BHT (butylated hydroxytoluene, $2.0 \mu \mathrm{L}$ ) were added to a plasma sample $(1.0 \mathrm{~mL})$ and mixed by vortex for 5 minutes. The mixture was centrifuged at 4,000 rpm for 10 minutes and then the supernatant was dried by nitrogen gas flow. Subsequently, $250 \mu \mathrm{L}$ of methanol was added to the dried sample and mixed by vortex. The solution was centrifuged at 4,000 rpm for 10 minutes and $5.0 \mu \mathrm{L}$ of the clear supernatant was injected into the LC-MS for analysis as previously described. This procedure was repeated for processing the tissue samples.

\section{Pharmacokinetic analysis and evaluation of targeting activity}

The concentration data of $\mathrm{YC} 1$ in plasma and tissue samples were pooled to obtain the mean concentration of $\mathrm{YCl}$. The pharmacokinetics of YC1 was described as a two-compartment open model and the pharmacokinetic parameters in the plasma and organs were obtained. The area under the concentrationtime curve (AUC) was calculated using the software program 3p97 (Mathematical Pharmacology Professional Committee of China; Shanghai, People's Republic of China). The mean residence time (MRT) was calculated by statistic moment program (DAS, Mathematical Pharmacology Professional Committee of China). To evaluate the targeting attribute of Lfmodified nanomicelles, MRT (mean residence time), overall targeting efficiency (TE), targeting index (TI), and relative overall targeting efficiency (RTE) were calculated as per the following equations (Equations 1-5) and compared with those of the $\mathrm{MC}$ and $\mathrm{YC}$ groups. ${ }^{30}$ In Equation 1, the denominator represents the total of YC1 exposure to all the tissues.

$$
\begin{aligned}
& \mathrm{TE}(\%)=\left(\mathrm{AUC}_{0 \rightarrow \infty}\right)_{i} / \sum_{i=1}^{\mathrm{n}}\left(\mathrm{AUC}_{0 \rightarrow \infty}\right)_{i} \times 100 \\
& \mathrm{TI}_{\mathrm{LF}}(\%)=\left(\mathrm{AUC}_{0 \rightarrow \infty}\right)_{\mathrm{LF} \mathrm{group}} /\left(\mathrm{AUC}_{0 \rightarrow \infty}\right)_{\mathrm{YCgroup}} \times 100 \\
& \mathrm{TI}_{\mathrm{MC}}(\%)=\left(\mathrm{AUC}_{0 \rightarrow \infty}\right)_{\mathrm{MC} \mathrm{group}} /\left(\mathrm{AUC}_{0 \rightarrow \infty}\right)_{\mathrm{YC} \mathrm{group}} \times 100 \\
& \mathrm{RTE}_{\mathrm{LF}}(\%)=\left(\mathrm{TE}_{\mathrm{LF} \text { group }}-\mathrm{TE}_{\mathrm{YC} \text { group }}\right) / \mathrm{TE}_{\mathrm{YC} \text { group }} \times 100 \\
& \mathrm{RTE}_{\mathrm{MC}}(\%)=\left(\mathrm{TE}_{\mathrm{MC} \text { group }}-\mathrm{TE}_{\mathrm{YC} \text { group }}\right) / \mathrm{TE}_{\mathrm{YCgroup}} \times 100
\end{aligned}
$$

\section{Statistical analysis}

Data were expressed as means $\pm \mathrm{SD}$. Statistical significance of the difference was assessed by one-way analysis of variance followed by post-hoc tests to determine the significance among groups in SPSS (v17.0; SPSS, Inc, Chicago, IL, USA). A $P$-value of $<0.05$ was considered significant.

\section{Results and discussion}

\section{Synthesis and characterization of SACD}

The synthetic route of SACD is briefly illustrated in Figure 2. In ${ }^{1} \mathrm{H}$ NMR spectra, several proton peaks at 1.0-2.5 ppm were assigned to the cholesteryl chain of cholesterol except $\mathrm{H} 3$ and H6. ${ }^{7}$ Moreover, the signals between 3.6 and 4.5 ppm could be considered as the methine protons of hexuronic acid residues of the alginate main chain ${ }^{26}$ and the $\mathrm{H} 3$ and $\mathrm{H} 6$ of the cholesteryl moiety. The single peak at 4.5-4.8 ppm was attributed to the methine protons of sodium alginate. The ${ }^{1} \mathrm{H}$ NMR spectrum of SACD (Figure 3C) displayed typical peaks of the protons on the alginate chain (Figure $3 \mathrm{~B}$ ) and the peaks (1.0-2.5 ppm) of protons on the carbons of cholesterol (Figure 3A). The difference between the spectra of SACD (Figure $3 \mathrm{C}$ ) and the physical mixture of sodium alginate and cholesterol (Figure 3D) indicated that cholesterol was successfully introduced onto sodium alginate to produce SACD. 

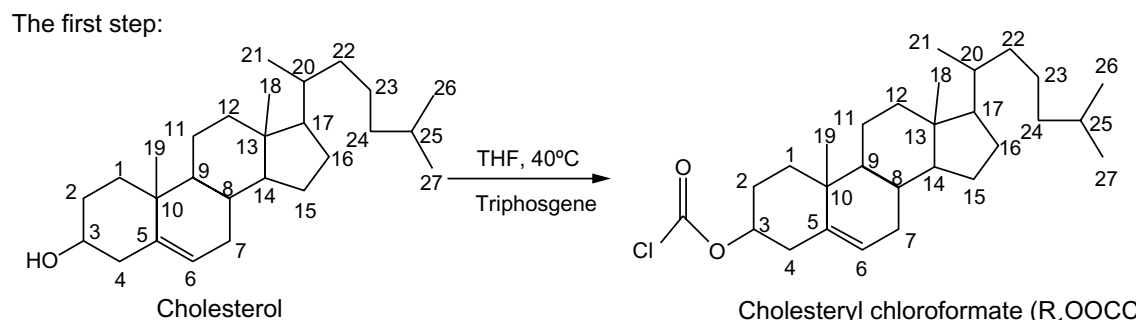

Cholesteryl chloroformate $\left(\mathrm{R}_{1} \mathrm{OOCCl}\right)$

The second step:

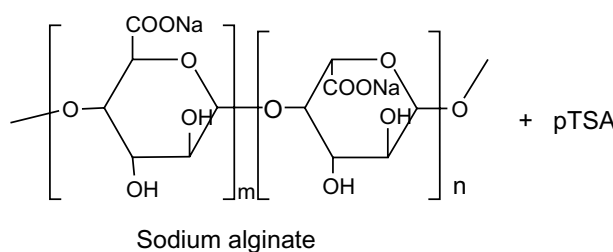

$\stackrel{\mathrm{DMF} / \mathrm{FA}, 50^{\circ} \mathrm{C} \sim 60^{\circ} \mathrm{C}}{\longrightarrow} \mathrm{R}_{2} \mathrm{COOH}$ (Alginic acid)

Sodium alginate

$\stackrel{+}{\text { DCC/DMAP }}$

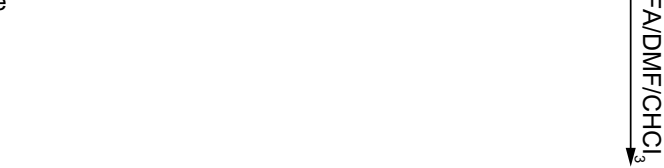

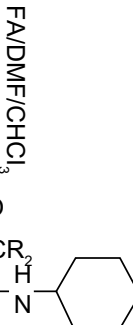

Figure 2 The synthetic route of SACD.

Abbreviation: $S A C D$, sodium alginate-cholesterol derivatives.

In the FT-IR spectrum (Figure 4), the absorption of double peaks between 1700 and $1600 \mathrm{~cm}^{-1}$ (Figure 4C) indicated the presence of two kinds of carbonyl groups from both carboxyl groups and newly formed ester bonds. However, in the FT-IR spectrum of sodium alginate (Figure 4B), only one peak was

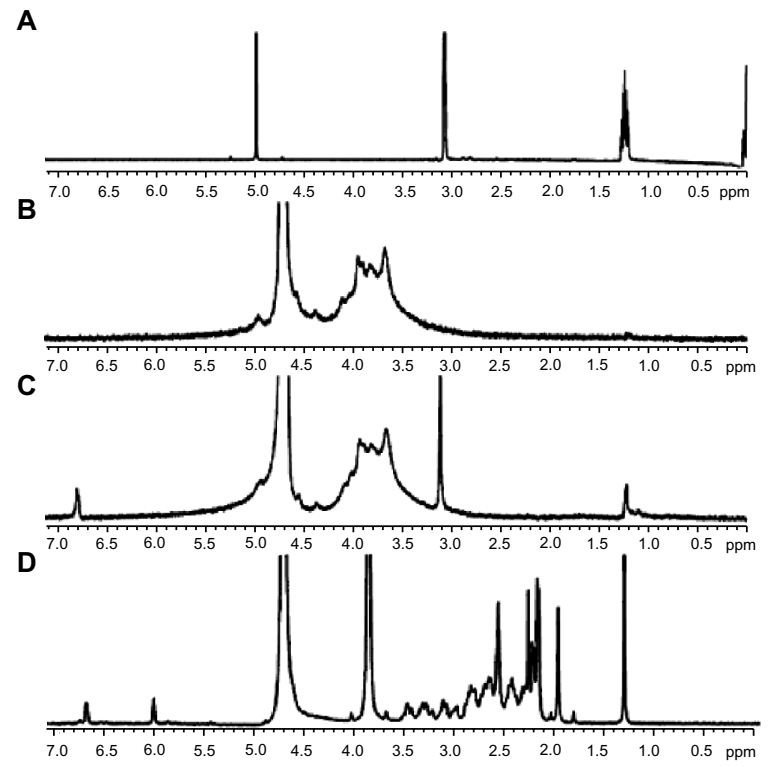

Figure 3 'H NMR spectra of $(\mathbf{A})$ cholesterol in $\mathrm{CCl}_{3} \mathrm{D}$; (B) sodium alginate in $\mathrm{D}_{2} \mathrm{O}$; (C) SACD in $\mathrm{D}_{2} \mathrm{O}$; and (D) mixture of cholesterol and sodium alginate in $\mathrm{CCl}_{3} \mathrm{D}$. Abbreviations: NMR, nuclear magnetic resonance; SACD, sodium alginatecholesterol derivatives; $\mathrm{CCl}_{3} \mathrm{D}$, deuterated chloroform; $\mathrm{D}_{2} \mathrm{O}$, heavy water. observed between 1700 and $1600 \mathrm{~cm}^{-1} .^{7}$ This distinction suggested that esterification between sodium alginate and cholesterol occurred.

\section{Characterization of Lf-SACD}

The electrophoretogram of Western blot analysis was shown in Figure 5. Column A supports the position of the
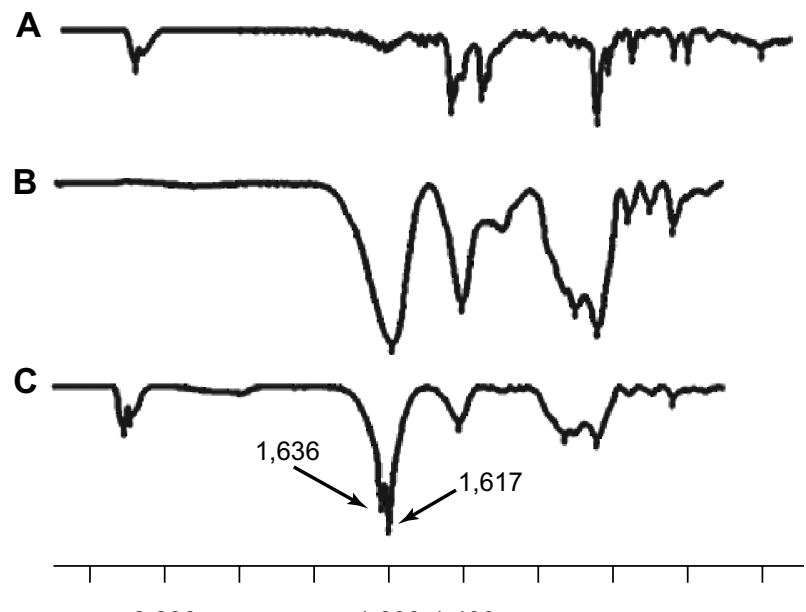

$2,400 \quad 2,2002,0001,8001,600 \quad 1,400 \quad 1,200 \quad 1,000 \quad 800 \quad 600$

Wavenumbers $\left(\mathrm{cm}^{-1}\right)$

Figure 4 FT-IR spectra of (A) cholesterol; (B) sodium alginate; and (C) SACD. Abbreviations: FT-IR, Fourier transform infrared; SACD, sodium alginate-cholesterol derivatives. 


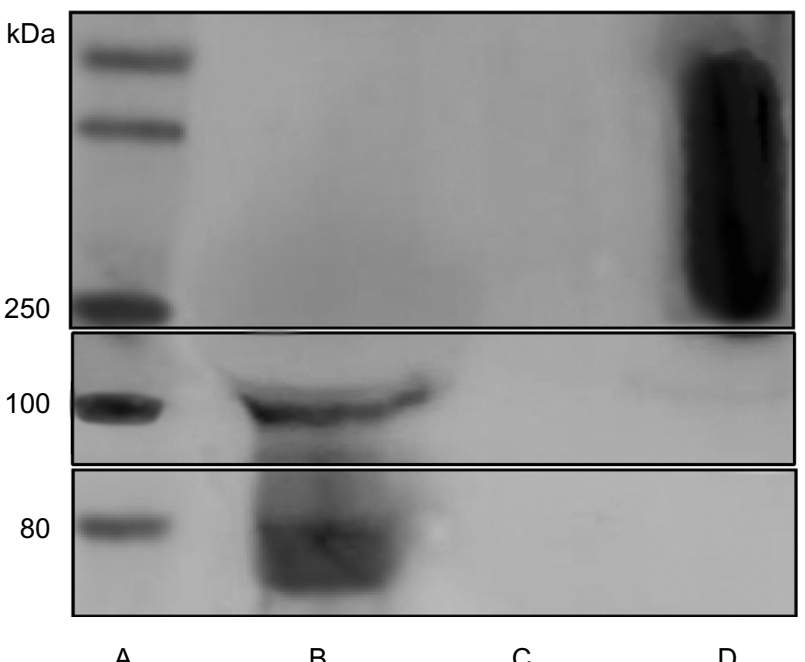

A

Figure 5 Electrophoretogram of Western blot analysis for (A) makers; (B) Lf (C) SACD; and (D) Lf-SACD.

Abbreviations: Lf, lactoferrin; SACD, sodium alginate-cholesterol derivatives.

markers $(\mathrm{kDa})$, while column $\mathrm{B}$ indicates that lactoferrin had a molecular weight of $70-80 \mathrm{kDa}$. Column $\mathrm{C}$ proved that the controlled group, SACD, was not immunogenic, while column D showed that the SACD was modified with lactoferrin, resulting in a molecular weight of $250-330 \mathrm{kDa}$ for Lf-SACD. Therefore, the IEM results confirmed that lactoferrin was successfully modified onto SACD.

\section{Characterization of self-assembly nanomicelles}

Particle sizes and zeta potentials of the unloaded and YC1loaded nanomicelles were investigated, respectively. The particle sizes of more than $70 \%$ of the $\mathrm{YC} 1$-unloaded nanomicelles ranged from 100 to $140 \mathrm{~nm}$ whereas the diameters of more than $70 \%$ of YC1-loaded nanomicelles were in the 140-180 nm range. Thus, the increased size of YC1-loaded nanomicelles demonstrates that YC1 molecules were encapsulated into the nanomicelles. In addition, the zeta potential was about $-100 \mathrm{mV}$ for both types of nanomicelles. This could be explained by the fact that the alginate ionized a large number of anionic - $\mathrm{COO}^{-}$carrying negative charges, and thus the whole system appeared to be negative.

The TEM images (Figure 6) of the Lf-SACD selfaggregates presented some core/shell-like particles with an approximate size of $100 \mathrm{~nm}$. The relatively uniform size distribution and approximately spherical and regular shape for the YC1-loaded nanomicelles confirmed that the existence of intramolecular and intermolecular hydrophobic interactions among the cholesteryl grafts made the Lf-SACD self-assemble into nanomicelles.

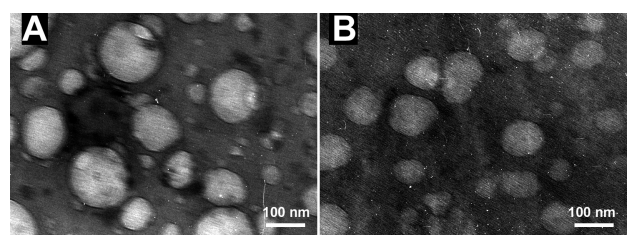

Figure 6 Microphotograph of Lf-SACD self-aggregates under transmission electron microscopy $(\times 80,000)$; (A) YCl-loaded; and (B) YCl-unloaded.

Abbreviations: Lf, lactoferrin; SACD, sodium alginate-cholesterol derivatives YCI, $5 \alpha$-cholestane-24-methylene-3 $\beta, 5 \alpha, 6 \beta$, 19-tetraol.

Shelf-life stability of YC1-loaded nanomicelles from lyophilization was studied by determining both the changes of the encapsulation efficiency and monitoring the particle size changes of nanomicelles after storage at $4^{\circ} \mathrm{C}$ for 15 days, 30 days, and 60 days. It appears that the nanomicelles displayed considerable stability and that only negligible changes of the particle size and the encapsulation efficiency were observed.

\section{CMC of SACD}

In this research, two synthesis schemes of SACD were performed, where the only difference between them was whether cholesterol was converted into cholesteryl chloroformate by triphosgene. It was revealed that the reaction between hydroxyl compounds and triphosgene would produce unstable chloroformate with high reactivity. ${ }^{31}$ Thus, the synthesis route in which cholesterol was pretreated by triphosgene was assumed to generate SACD with a higher grafting ratio and lower CMC.

When cholesterol was not pretreated (Figure 7A) or pretreated (Figure 7B) by triphosgene for the synthesis of SACD, the CMC values of the obtained nanomicelles were about $2.0 \mathrm{mg} / \mathrm{L}$ and $0.8 \mathrm{mg} / \mathrm{L}$, respectively. According to Yang et al, ${ }^{7}$ there were several types of intra- and inter-molecular interactions controlling SACD to selfassemble, such as the hydrophobic interaction among cholesteryl grafts, the hydrophilic bonding between the backbones and water molecules as well as among sodium alginate backbones themselves. Amongst these interactions, those between cholesteryl grafts played the most important role. In other words, more cholesterol connected with sodium alginate may generate a lower CMC value for SACD. Accordingly, the nanomicelles obtained from the pretreated cholesterol possessed a lower CMC value (Figure 7B), which suggested that the SACD had a higher cholesterol grafting ratio. It is important and meaningful in practice, as a lower CMC value for nanomicelles guarantees their use in very dilute media, such as in body fluids. 


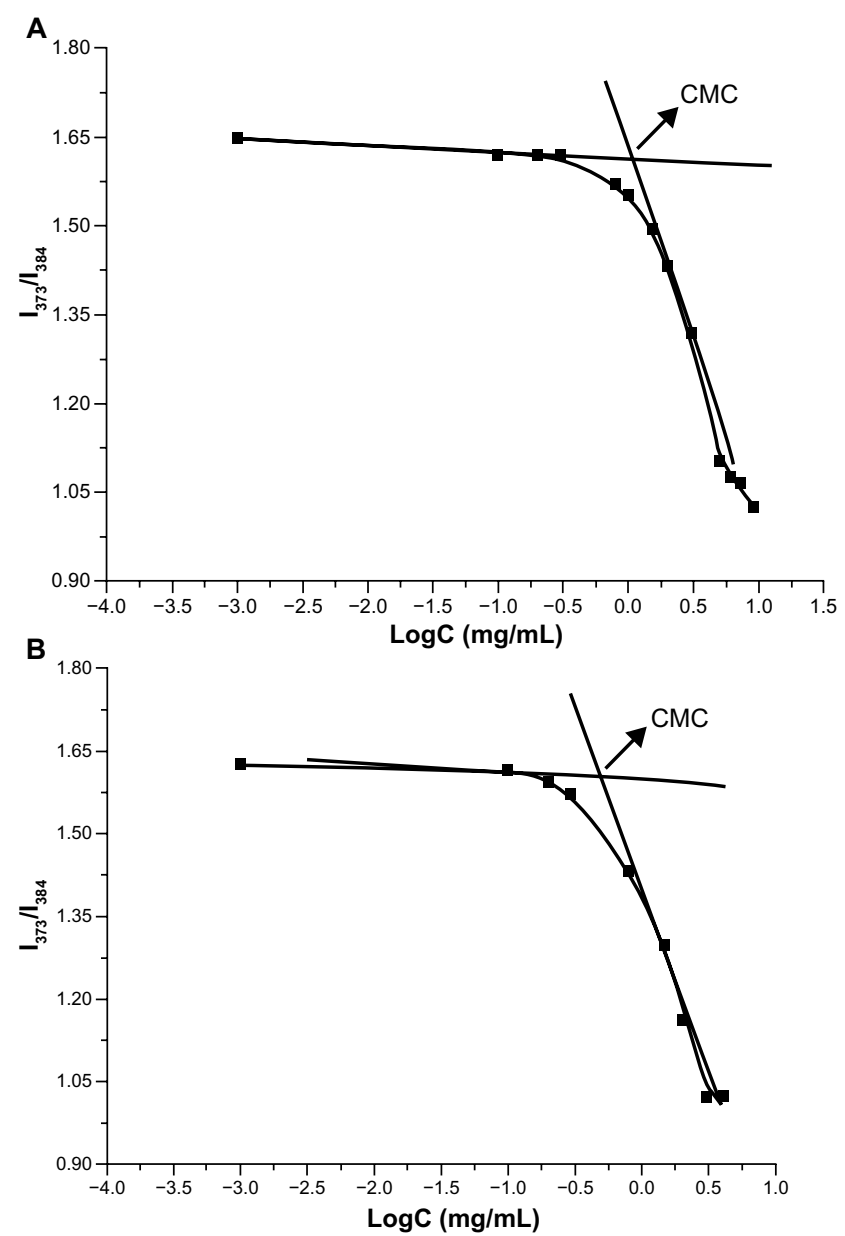

Figure 7 Plots of $I_{373} / I_{384}$ ratio as a function of logarithm concentration of SACD with cholesterol not pretreated $(\mathbf{A})$ or pretreated $(\mathbf{B})$ by triphosgene.

Abbreviations: SACD, sodium alginate-cholesterol derivatives; CMC, critical micelle concentration.

\section{Drug loading and the relationship between the grafting ratio and encapsulation efficiency}

SACDs with distinct grafting ratios were fabricated from different ratios of cholesterol and sodium alginate. Figure 8 shows that encapsulation efficiency improves with an increase in grafting ratio, suggesting a positive association between them. It was reported that the encapsulation efficiency of nanomicelles depends on the structure of drugs and the core block of the polymers. If the hydrophobic core of the polymers is similar to the structure of the loaded drugs, then the encapsulation efficiency would increase by thousands-fold. ${ }^{32}$ In this study, $\mathrm{YC} 1$ is one kind of oxysterol with the structure of a steroid that is identical to the parent nucleus of cholesterol. Therefore, with an increase in the cholesteryl grafting ratio, the intermolecular interaction between cholesteryl and YC1 would build up and YC1

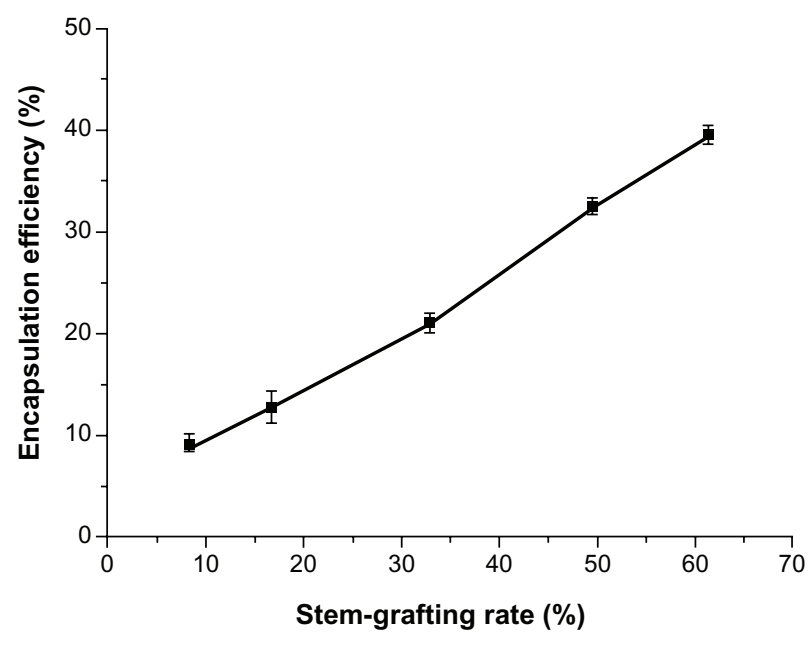

Figure 8 Relationship between the grafting ratio and the drug loading $(n=3)$.

could be entrapped into the inner core more easily and with more stability. It should be emphasized that Lf-SACD nanomicelles showcased higher drug loading for hydrophobic drugs when compared with other amphiphilic polymer-based nanocarriers. ${ }^{33-35}$

\section{In vitro drug release}

Figure 9 shows that the accumulative release rate of $\mathrm{YC} 1$ from the nanomicelles could reach more than $80 \%$ in the first 12 hours in the releasing medium of $6 \% \mathrm{HP}-\beta-\mathrm{CD}$ and that no dumping effect was observed in the first 5 hours. The release behavior could last for 12 hours, implying that $\mathrm{YC} 1$ was well encapsulated into the nanomicelles. Thus, the nanomicelles may prolong the retention time of $\mathrm{YC} 1$ in the brain, extending

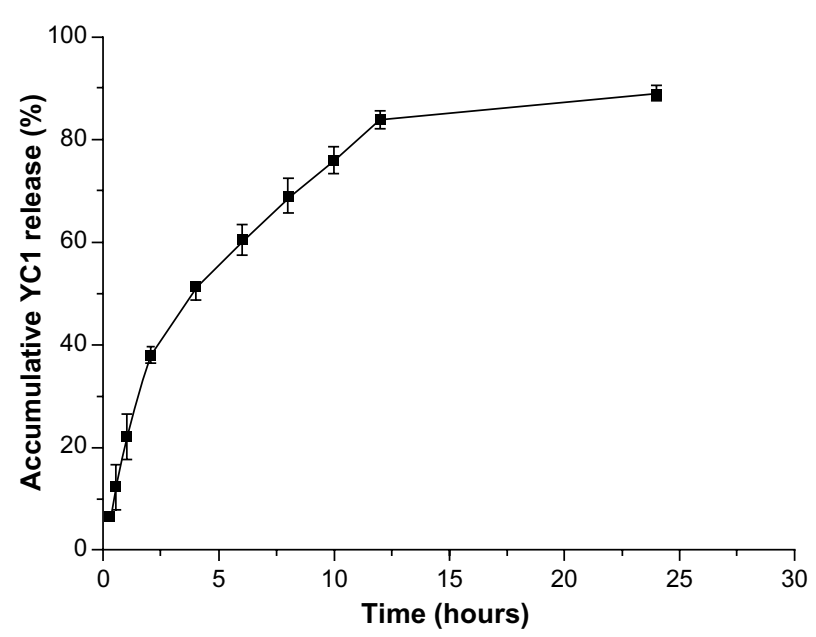

Figure 9 Release profile of $Y C I$ from SACD nanomicelles in $6 \% \mathrm{HP}-\beta-\mathrm{CD}$ at $37^{\circ} \mathrm{C}$ $(n=3)$.

Abbreviations: $\mathrm{SACD}$, sodium alginate-cholesterol derivatives $\mathrm{YCI}, 5 \alpha$-cholestane24-methylene-3 $\beta, 5 \alpha, 6 \beta$, 19-tetraol. 
Table I Models and equations of $\mathrm{YCI}$ releasing from nanomicelles in $6 \% \mathrm{HP}-\beta-\mathrm{CD}$ at $37^{\circ} \mathrm{C}$

\begin{tabular}{lll}
\hline Model & Equation & $\boldsymbol{r}^{2}$ \\
\hline Zero-order & $y=3.4191 \mathrm{x}+27.247$ & 0.7221 \\
First-order & $\mathrm{y}=-0.0897 \mathrm{x}+4.3176$ & 0.8882 \\
Higuchi & $\mathrm{y}=20.266 \mathrm{x}+4.965$ & 0.9186 \\
Ritger-Peppas & $\mathrm{y}=0.5943 \mathrm{x}+2.9473$ & 0.9446 \\
\hline
\end{tabular}

Abbreviation: $\mathrm{YCI}, 5 \alpha$-cholestane-24-methylene-3 $\beta, 5 \alpha, 6 \beta$, 19-tetraol.
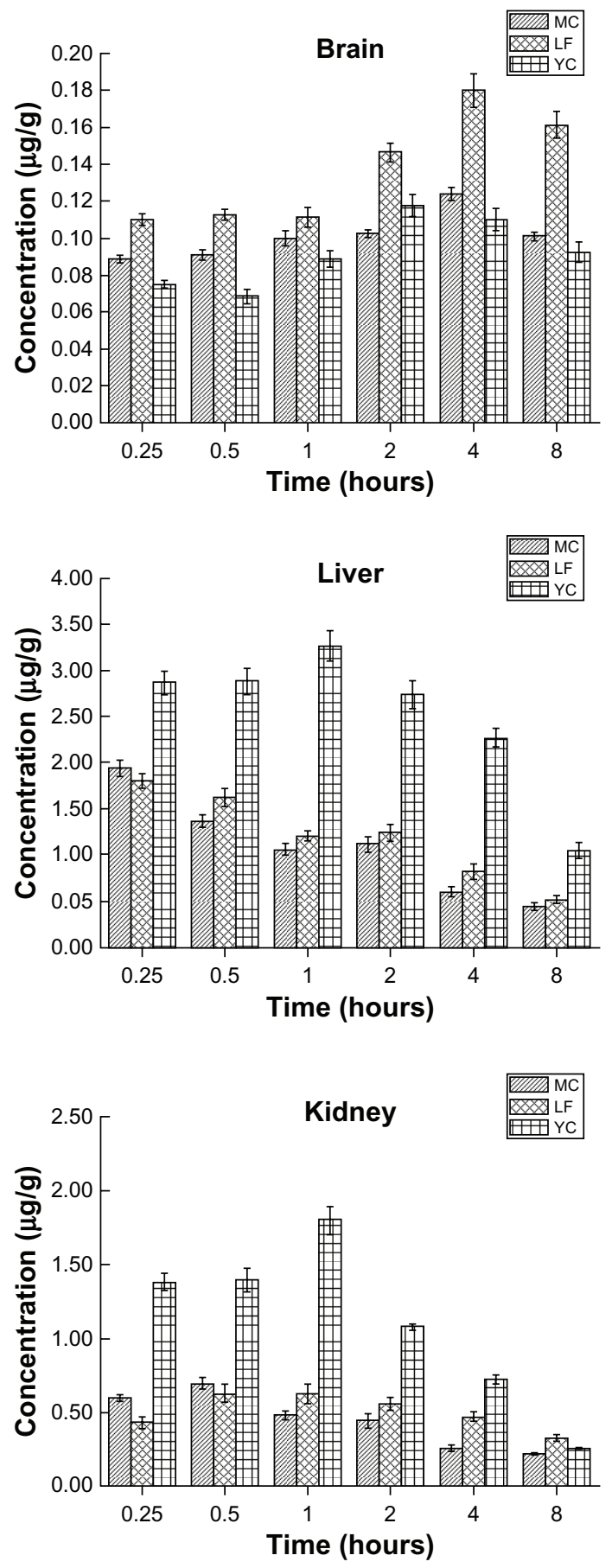

the potential neuroprotection time. Furthermore, different release mechanisms were proposed for $\mathrm{YC} 1$-loaded nanomicelles releasing in HP- $\beta$-CD. Based on the values of $r^{2}$ in Table 1, the release from the nanomicelles most likely fits to the Ritger-Peppas equation, which suggests that the release mechanism was due to the joint effects of drug diffusion and skeleton dissolution.
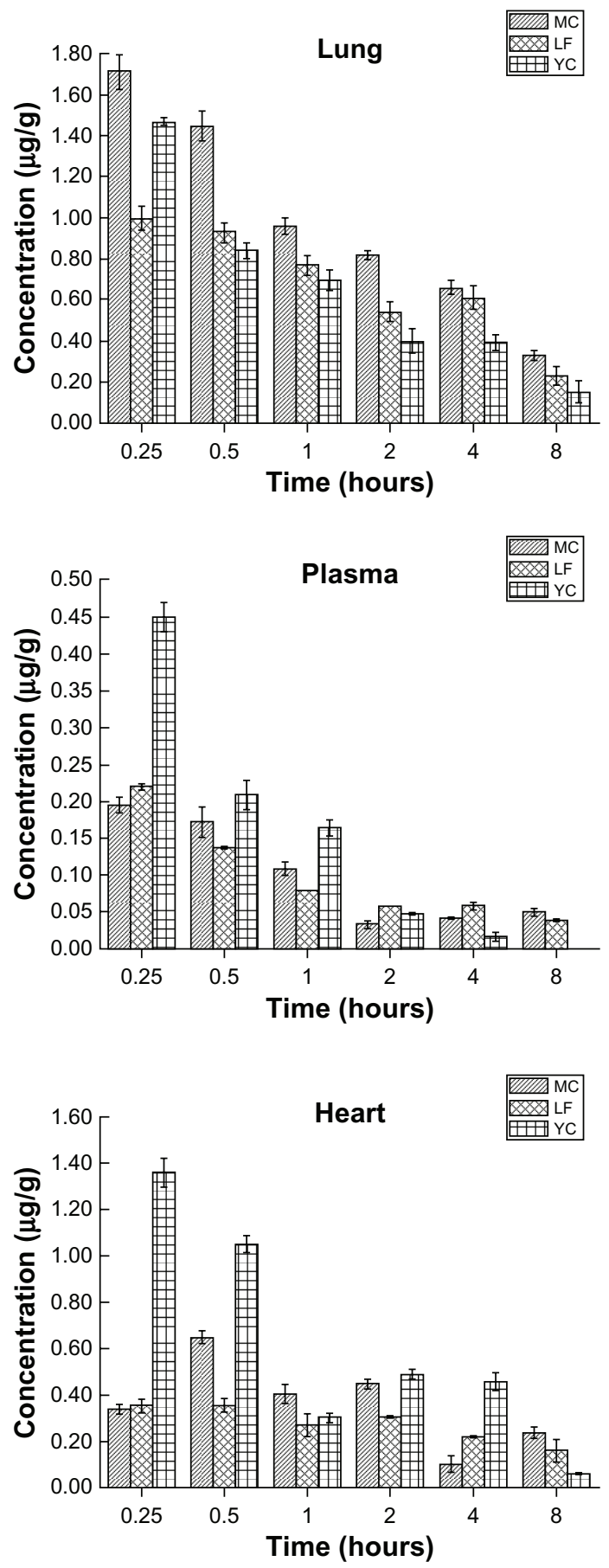

Figure 10 Concentration of $\mathrm{YCI}$ in plasma and tissues after iv administration of YCI-loaded nanomicelles (MC), YCI-loaded Lf-modified nanomicelles (LF), and YCI solution (YC) $(n=5)$.

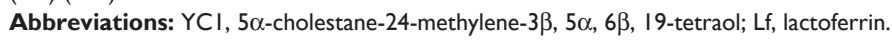




\section{Biodistribution of drugs in mice}

The distribution of $\mathrm{YC} 1$ in the plasma and tissues of mice after iv administration of YC1-loaded Lf-modified nanomicelles (LF), YC1-loaded nanomicelles (MC), and YC1 solution (YC) separately was investigated. The results (Figure 10) indicate that the $\mathrm{YC} 1$ concentration in the brain for the $\mathrm{LF}$ group was significantly higher than those for the other two groups; the concentration in the brain reached its maximum about 4 hours after injection. In some excretory organs such as liver and kidney, $\mathrm{YC} 1$ concentration of the $\mathrm{MC}$ and $\mathrm{LF}$ groups was much lower than that of the YC group. This might be ascribed to the fact that nanomicelles can attenuate the elimination and extend the residence time of $\mathrm{YC} 1$, and thus the drug could be targeted to a specific organ with long circulating characteristics.

\section{Pharmacokinetic analysis and evaluation of targeting activity}

Based on the pharmacokinetic analysis and evaluation of targeting activity for $\mathrm{YC} 1$ in mice after iv administration of YC1 solution, YC1-loaded modified or non-modified nanomicelles, clear differences between these groups were found in the YC1 concentrations in organs (Figure 10), the pharmacokinetic parameters (Tables 2 and 3), and the TE (Tables 4 and 5; Figure 11). In comparison with YC and MC groups, the brain TE of the LF group was greatly improved. For example, the brain TE was enhanced

Table 2 Pharmacokinetic parameters of $\mathrm{YCl}$ in mice after iv administration of YC group, MC group, and LF group

\begin{tabular}{llll}
\hline Parameters & YC group & MC group & LF group \\
\hline $\mathrm{A}(\mu \mathrm{g} / \mathrm{mL})$ & $0.46 \mathrm{I}$ & 0.189 & 0.322 \\
$\mathrm{~B}(\mu \mathrm{g} / \mathrm{mL})$ & $0.07 \mathrm{I}$ & 0.069 & 0.067 \\
$\alpha\left(\mathrm{h}^{-1}\right)$ & 1.645 & 1.209 & 2.927 \\
$\beta\left(\mathrm{h}^{-1}\right)$ & 0.156 & 0.237 & 0.066 \\
$t_{1 / 2} \alpha(\mathrm{h})$ & 0.421 & 0.573 & 0.237 \\
$t_{1 / 2} \beta(\mathrm{h})$ & $4.45 \mathrm{I}$ & 2.920 & 10.515 \\
$K_{21}\left(\mathrm{~h}^{-1}\right)$ & 0.354 & 0.498 & 0.559 \\
$K_{10}\left(\mathrm{~h}^{-1}\right)$ & 0.724 & 0.576 & 0.345 \\
$K_{12}\left(\mathrm{~h}^{-1}\right)$ & 0.723 & 0.372 & 2.089 \\
$\mathrm{AUC}(\mu \mathrm{g} \cdot \mathrm{h} / \mathrm{g})$ & 0.734 & 0.448 & 1.128 \\
$\mathrm{MRT}(\mathrm{h})$ & 7.017 & 5.030 & 15.483 \\
$\mathrm{Cl}(\mathrm{mL} / \mathrm{h})$ & 217.927 & 357.200 & 141.868 \\
$\mathrm{C}_{0}(\mu \mathrm{g} / \mathrm{mL})$ & 0.531 & 0.258 & 0.389 \\
$\mathrm{Vc}(\mathrm{mL})$ & 301.195 & 620.509 & 411.394 \\
$\mathrm{~V}(\mathrm{~mL})$ & 1399.821 & 1505.134 & 2152.617 \\
\hline $\mathrm{Notes}$ & &
\end{tabular}

Notes: $\mathrm{LF}$ group $=\mathrm{YCI}$-loaded Lf-modified nanomicelles; $\mathrm{MC}$ group $=\mathrm{YCI}$-loaded nanomicelles without conjugating $L f ; Y C$ group $=Y C I$ solution.

Abbreviations: Lf, lactoferrin; $\mathrm{YCI}, 5 \alpha$-cholestane-24-methylene- $3 \beta, 5 \alpha, 6 \beta$, 19-tetraol; iv, intravenous.
Table 3 Pharmacokinetic parameters of $\mathrm{YCl}$ of $\mathrm{YC}$ group, $\mathrm{MC}$ group, and LF group in brain tissue

\begin{tabular}{lllll}
\hline Parameters & $\begin{array}{l}\text { AUC }_{0 \rightarrow \infty} \\
(\boldsymbol{\mu} \cdot \mathbf{h} / \mathbf{g})\end{array}$ & $\begin{array}{l}\text { MRT } \\
(\mathbf{h})\end{array}$ & $\begin{array}{l}\boldsymbol{t}_{1 / 2} \\
(\mathbf{h})\end{array}$ & $\begin{array}{l}\text { VRT } \\
\mathbf{( h \cdot h )}\end{array}$ \\
\hline YC group & 2.8 & 3.9 & 2.70 & 6.1 \\
MC group & 4.1 & 4.1 & 2.84 & 7.0 \\
LF group & 7.1 & 4.3 & 2.99 & 6.2 \\
\hline
\end{tabular}

Notes: LF group $=$ YCI-loaded Lf-modified nanomicelles; $\mathrm{MC}$ group $=\mathrm{YCI}$-loaded nanomicelles without conjugating Lf; $Y C$ group $=\mathrm{YCI}$ solution.

Abbreviations: AUC, area under the curve; MRT, mean residence time; VRT, variance of mean residence time; h, hour, $\mathrm{YCl}, 5 \alpha$-cholestane-24-methylene-3 $3 \beta, 5 \alpha$, $6 \beta$, 19-tetraol; Lf, lactoferrin.

from $6.9 \%$ for the YC group to $18.7 \%$ for the LF group (Table 4), and the RTE of the LF group was about two-fold greater than that of the MC group (Table 5). Conclusively, Lf-modified nanomicelles played a rather significant role in enhancing the brain TE of YC1. The noticeable enhancement of YC1 CNS distribution through the delivery system of Lf-modified nanomicelles was mainly derived from the following reasons. A large amount of YC1 solution was distributed in the liver, lung, and kidney, and was metabolized and excreted quickly; however, the YC1-loaded nanomicelles had a significantly lower YC1 concentration in these organs, and thus YC1 was eliminated at a slower rate. This phenomenon could be attributed to the fact that nanomicelles could remarkably encapsulate the steroidal YC1 to the hydrophobic inner core owing to their structural resemblance and simultaneously endow $\mathrm{YC} 1$ with the properties of sustained release, targeting, and lower accessibility to metabolic enzymes. On the other hand, there were measurable differences between the $\mathrm{MC}$ group and LF group in brain AUC, TI, and RTE, showing that lactoferrin enhanced the cerebral targeted delivery of YC1. This proved that lactoferrin receptor-mediated endocytosis actually assisted Lf-modified YC1-containing nanomicelles crossing the BBB. ${ }^{27}$ In summary, these combining factors contributed to the elevation of YC1 in CNS distribution and reduction in other main tissues.

Interestingly, the $\mathrm{YC} 1$ content of the $\mathrm{MC}$ group in the lungs was much higher than that of the YC group, showing a passive

Table 4 Overall TE of LF, MC, and YC groups

\begin{tabular}{lllllll}
\hline Tissue & Plasma & Heart & Liver & Lung & Kidney & Brain \\
\hline YC group & I.2 & 8.4 & 57.2 & 9.4 & 27.1 & 6.9 \\
MC group & 3.4 & II.I & 27.3 & 21.7 & 24.0 & 12.5 \\
LF group & 2.1 & 8.2 & 28.1 & 13.6 & 20.5 & 18.7 \\
\hline
\end{tabular}

Notes: LF group $=$ YCI-loaded Lf-modified nanomicelles; $\mathrm{MC}$ group $=\mathrm{YCI}$-loaded nanomicelles without conjugating $L f ; Y C$ group $=\mathrm{YCI}$ solution.

Abbreviations: TE, targeting efficiency; $\mathrm{YCl}, 5 \alpha$-cholestane-24-methylene- $3 \beta, 5 \alpha$, $6 \beta$, 19-tetraol; Lf, lactoferrin. 
Table $5 \mathrm{TI}$ and RTE of LF and MC groups

\begin{tabular}{lllllll}
\hline Tissue & Plasma & Heart & Liver & Lung & Kidney & Brain \\
\hline $\mathrm{TI}_{\mathrm{LF}}(\%)$ & 161.9 & 91.9 & 46.8 & 137.8 & 70.1 & 255.9 \\
$\mathrm{TI}_{\mathrm{MC}}(\%)$ & 217.6 & 106.2 & 38.5 & 186.4 & 69.7 & 146.4 \\
$\mathrm{RTE}_{\mathrm{LF}}(\%)$ & 75.0 & -0.7 & -49.5 & 48.9 & -24.2 & 176.6 \\
$\mathrm{RTE}_{\mathrm{MC}}(\%)$ & 176.6 & 35.1 & -51.0 & 137.0 & -11.3 & 86.1 \\
\hline
\end{tabular}

Notes: LF group = YCI-loaded Lf-modified nanomicelles; $M C$ group $=$ YCI-loaded nanomicelles without conjugating $L f$.

Abbreviations: RTE, relative overall targeting efficiency; TI, targeting index; $\mathrm{YCl}$, $5 \alpha$-cholestane-24-methylene-3 $3,5 \alpha, 6 \beta$, 19-tetraol; Lf, lactoferrin.

targeting effect of YC1-loaded unmodified nanomicelles in the lungs (Table 4 and Figure 10). This phenomenon might be accounted for by the dual reasons that a) the lung is a highly perfused organ by circulating blood and b) the cells in the reticuloendothelial system (RES) of the lung tissue phagocytosed the nanomicelles when the particle size was in the range of 30-150 nm. ${ }^{36}$ However, the LF-modified nanomicelles showed much lower $\mathrm{YC} 1$ concentration in the lungs due to the ability of active targeting of the preparation.

Considering the significant differences in brain AUC, TE, TI, and RTE among the three groups, it is logical to surmise that the mean residence time (MRT) and $t_{1 / 2}$ of the LF group should extend far more than those of the $\mathrm{MC}$ and YC group. However, the retention time of $\mathrm{YC} 1$ in the brain for the $\mathrm{LF}$ group was not as long as expected. This might be ascribed mainly to two reasons: a) the blood sampling time was not long enough and there was still plenty of $\mathrm{YC} 1$ remaining in the brain after 8 hours (Figure 10); and b) the amount of lactoferrin successfully conjugated onto SACD was insufficient. Since only $125 \mu \mathrm{g}$ of lactoferrin was added to $5.0 \mathrm{mg}$ of SACD to react, the active brain-targeting effect was limited by

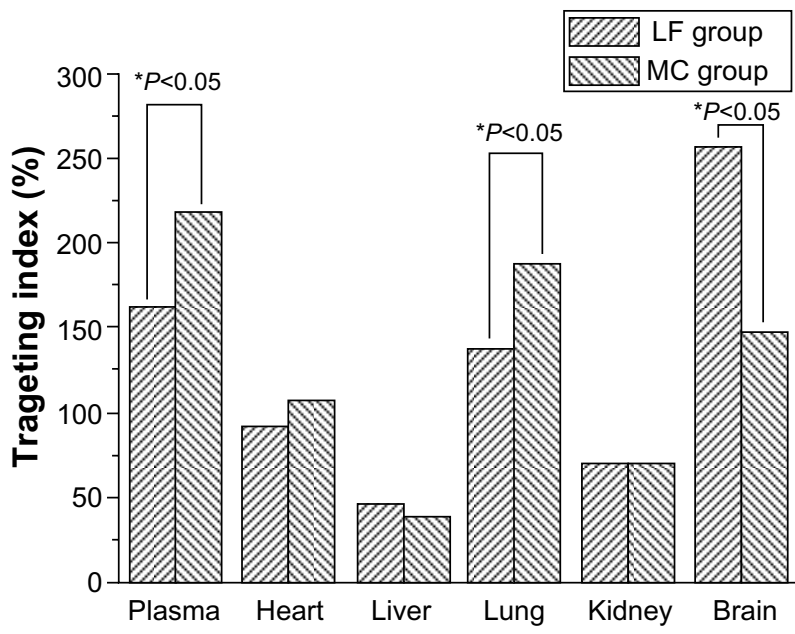

Figure II $\mathrm{TI}$ of $\mathrm{YCl}$-loaded nanomicelles $(\mathrm{MC})$ and $\mathrm{YCI}$-loaded Lf-modified nanomicelles (LF) in different tissues.

Abbreviations: TI, targeting index; $\mathrm{YCI}, 5 \alpha$-cholestane-24-methylene- $3 \beta, 5 \alpha, 6 \beta$, 19-tetraol; Lf, lactoferrin. the inadequate number of lactoferrin ligands on the surfaces of nanomicelles.

\section{Conclusion}

YC1-loaded nanomicelles, self-assembling from the amphiphilic polymer Lf-SACD, have been successfully prepared for drug delivery across the BBB. Firstly, one of the most significant features of the delivery system was the hydrophobic inner core that can accommodate a relatively large amount of steroidal YC1 owning to the "like dissolves like' rule. Moreover, the drug distribution in mice showed that the Lf-modified nanomicelles exhibited the properties of sustained release, drug protection, and a brain-targeting effect. The presence of lactoferrin on the surface of the nanomicelles promoted the accumulation of $\mathrm{YC} 1$ in the brain, achieving the goal of increasing the cerebral concentration of YC1. Finally, for the first time, cholesterol was chloroformylated in this research before reacting with sodium alginate, resulting in amphiphilic polymer SACD with a higher grafting ratio. This further led to increasing the drug loading, reducing the $\mathrm{CMC}$ value, and enhancing the stability of micelles in dilute solutions. In short, it can be concluded that Lf-modified sodium alginate-cholesterol nanomicelles show a potential to be a highly efficient drug carrier of hydrophobic therapeutic agents for brain targeting.

\section{Acknowledgments}

Financial support from the Guangdong Natural Science Foundation (9451008901002309) is gratefully acknowledged.

\section{Disclosure}

The authors report no conflicts of interest in this work.

\section{References}

1. Xu S-B, Wang Z-L, Liu Q, Li R-S. Anti-inflammation actions of soft coral cholestane. Tropic Oceanology. 1999(2):27-32.

2. Guangmei Yan JZ, et al; inventors. Application of oceanic steroidal compounds in preparing neuron lesion theraputic agents. China patent CN200610036926.

3. Branco MC, Schneider JP. Self-assembling materials for therapeutic delivery. Acta Biomater. 2009;5(3):817-831.

4. BE Duneau A, Saulnier P, Benoit JP. Active targeting of brain tumors using nanocarriers. Biomaterials. 2007;28(33):4947-4967.

5. Schmid R. Recent advances in the description of the structure of water, the hydrophobic effect, and the like-dissolves-like rule. Highlights in Solute-Solvent Interactions. Berlin: Springer; 2002 59-90.

6. Robitzer M, David L, Rochas C, Renzo FD, Quignard F. Nanostructure of calcium alginate aerogels obtained from multistep solvent exchange route. Langmuir. 2008;24(21):12547-12552.

7. Yang L, Zhang B, Wen L, Liang Q, Zhang LM. Amphiphilic cholesteryl grafted sodium alginate derivative: Synthesis and self-assembly in aqueous solution. Carbohydr Polym. 2007;68(2):218-225. 
8. Ying X, Wen H, Lu W-L, et al. Dual-targeting daunorubicin liposomes improve the therapeutic efficacy of brain glioma in animals. $J$ Control Release. 2010;141(2):183-192.

9. Pardridge WM. Crossing the blood-brain barrier: are we getting it right? Drug Discov Today. 2001;6(1):1-2.

10. Patel MM, Goyal BR, Bhadada SV, Bhatt JS, Amin AF. Getting into the brain: approaches to enhance brain drug delivery. CNS Drugs. 2009;23(1):35-58.

11. Visser CC, Voorwinden LH, Crommelin DJ, Danhof M, de Boer AG. Characterization and modulation of the transferrin receptor on brain capillary endothelial cells. Pharm Res. 2004;21(5):761-769.

12. Bickel U, Yoshikawa T, Pardridge WM. Delivery of peptides and proteins through the blood-brain barrier. Adv Drug Deliv Rev. 2001; 46(1-3):247-279.

13. Pardridge WM, Eisenberg J, Yang J. Human blood-brain barrier transferrin receptor. Metabolism. 1987;36(9):892-895.

14. Jefferies WA, Brandon MR, Hunt SV, Williams AF, Gatter KC, Mason DY. Transferrin receptor on endothelium of brain capillaries. Nature. 1984;312(5990):162-163.

15. Letchford K, Burt H. A review of the formation and classification of amphiphilic block copolymer nanoparticulate structures: micelles, nanospheres, nanocapsules and polymersomes. Eur J Pharm Biopharm. 2007;65(3):259-269.

16. Baker EN, Baker HM, Kidd RD. Lactoferrin and transferrin: functional variations on a common structural framework. Biochem Cell Biol. 2002;80(1):27-34.

17. Huang R, Ke W, Liu Y, Jiang C, Pei Y. The use of lactoferrin as a ligand for targeting the polyamidoamine-based gene delivery system to the brain. Biomaterials. 2008;29(2):238-246.

18. Talukder MJ, Takeuchi T, Harada E. Receptor-mediated transport of lactoferrin into the cerebrospinal fluid via plasma in young calves. $J$ Vet Med Sci. 2003;65(9):957-964.

19. Fillebeen C, Descamps L, Dehouck MP, et al. Receptor-mediated transcytosis of lactoferrin through the blood-brain barrier. J Biol Chem. 1999;274(11):7011-7017.

20. Elfinger M, Maucksch C, Rudolph C. Characterization of lactoferrin as a targeting ligand for nonviral gene delivery to airway epithelial cells. Biomaterials. 2007;28(23):3448-3455.

21. Grau AJ, Willig V, Fogel W, Werle E. Assessment of plasma lactoferrin in Parkinson's disease. Mov Disord. 2001;16:131-134.

22. Terent A, Hallgren R, Venge P, Bergstrom K. Lactoferrin, lysozyme, and beta 2-microglobulin in cerebrospinal fluid. Elevated levels in patients with acute cerebrovascular lesions as indices of inflammation. Stroke. $1981 ; 12(1): 40-46$
23. Cipolla MJ, Crete R, Vitullo L, Rix RD. Transcellular transport as a mechanism of blood-brain barrier disruption during stroke. Front Biosci. 2004;9:777-785.

24. Chen Y, Liu L. Modern methods for delivery of drugs across the bloodbrain barrier. Adv Drug Deliv Rev. 2012;64(7):640-665.

25. Heggelund A, Undheim K. Descladinosyl erythromycin in phosgeneassisted cyclic 3,6-ether formation. Tetrahedron Lett. 2008;49(39): 5569-5571.

26. Liu L, Guo K, Lu J, et al. Biologically active core/shell nanoparticles self-assembled from cholesterol-terminated PEG-TAT for drug delivery across the blood-brain barrier. Biomaterials. 2008;29(10):1509-1517.

27. Hu KL, Li JW, Shen YH, et al. Lactoferrin-conjugated PEG-PLA nanoparticles with improved brain delivery: in vitro and in vivo evaluations. J Control Release. 2009;134:55-61.

28. Sethuraman VA, Bae YH. TAT peptide-based micelle system for potential active targeting of anti-cancer agents to acidic solid tumors. J Control Release. 2007;118(2):216-224.

29. Goddard ED, Turro NJ, Kuo PL, Ananthapadmanabhan KP. Fluorescence probes for critical micelle concentration determination. Langmuir. 1985;1(3):352-355.

30. Wang JX, Sun X, Zhang ZR. Enhanced brain targeting by synthesis of $3^{\prime}, 5^{\prime}$-dioctanoyl-5-fluoro-2'-deoxyuridine and incorporation into solid lipid nanoparticles. Eur J Pharm Biopharm. 2002;54(3): 285-290.

31. Goren Z, Heeg MJ, Mobashery S. Facile chloride substitution of activated alcohols by triphosgene: application to cephalosporin chemistry. J Org Chem. 1991;56(25):7186-7188.

32. Liu JB, Xiao YH, Allen C. Polymer-drug compatibility: a guide to the development of delivery systems for the anticancer agent, ellipticine. J Pharm Sci. 2004;93:132-143.

33. Vassiliou AA, Papadimitriou SA, Bikiaris DN, Mattheolabakis G, Avgoustakis K. Facile synthesis of polyester-PEG triblock copolymers and preparation of amphiphilic nanoparticles as drug carriers. J Control Release. 2010;148(3):388-395.

34. Wang J, del Rosario LS, Demirdirek B, Bae A, Uhrich KE. Comparison of PEG chain length and density on amphiphilic macromolecular nanocarriers: self-assembled and unimolecular micelles. Acta Biomater. 2009;5(3):883-892.

35. Memisoglu E, Bochot A, Sen M, Duchene D, Hincal AA. Non-surfactant nanospheres of progesterone inclusion complexes with amphiphilic beta-cyclodextrins. Int J Pharm. 2003;251(1-2):143-153.

36. Veiseh O, Gunn JW, Zhang M. Design and fabrication of magnetic nanoparticles for targeted drug delivery and imaging. Adv Drug Deliv Rev. 2010;62(3):284-304
International Journal of Nanomedicine

\section{Publish your work in this journal}

The International Journal of Nanomedicine is an international, peerreviewed journal focusing on the application of nanotechnology in diagnostics, therapeutics, and drug delivery systems throughout the biomedical field. This journal is indexed on PubMed Central, MedLine, CAS, SciSearch $\AA$, Current Contents ${ }^{\circledR} /$ Clinical Medicine,

\section{Dovepress}

Journal Citation Reports/Science Edition, EMBase, Scopus and the Elsevier Bibliographic databases. The manuscript management system is completely online and includes a very quick and fair peer-review system, which is all easy to use. Visit http://www.dovepress.com/ testimonials.php to read real quotes from published authors. 